\title{
Optimal Capacity and Operating Strategy for Providing Frequency Containment Reserve with Batteries and Power-to-Heat
}

\author{
Uli Schlachter, Alena Worschech, Theys Diekmann, Benedikt Hanke, Karsten von Maydell \\ DLR Institute of Networked Energy Systems, Carl-von-Ossietzky-Straße 15, 26129 Oldenburg, Germany
}

\begin{abstract}
Batteries provide growing amounts of Frequency Containment Reserve (FCR) in the German electricity market. We examine the ideal battery capacity as well as the optimal state of charge (SoC) setpoint for provision of FCR following a profit maximization approach. While a lot of research neglects taxes, their huge influence on the results is considered. In addition to stand-alone battery systems, we also consider hybrid systems, combining a battery storage with a Powerto-Heat (PtH) module. The hybrid system has two variants: In one variant energy is sold when the battery is fully charged, while in the other one the $\mathrm{PtH}$ module is used. Based on historical frequency and market data for one year, the performance of the different system variants is evaluated via a simulation. 2048 different combinations of battery capacity and SoC setpoint are examined. The performance of each configuration is evaluated with the net present value (NPV) method, allowing an economic comparison. The results show a financial advantage for the hybrid systems which sell energy when the battery is fully charged. The system configuration with the best NPV is a hybrid system with a battery capacity of $480 \mathrm{kWh}$ and an SoC setpoint of $98 \%$. The optimal battery capacity of all considered variants has a lower capacity to power ratio than the usual ratio of $1 \mathrm{MWh}$ to $1 \mathrm{MW}$, assumed in most other studies. Nonoptimal battery capacities have a stronger negative effect on the economic efficiency of the systems than non-optimal $\mathrm{SoC}$ setpoints. The results emphasise that the battery capacity is a critical investment decision.
\end{abstract}

Keywords: Primary Control Reserve, Degrees of Freedom, Net Present Value approach, Battery capacity sizing, SoC management

\section{Introduction}

On the way to a $\mathrm{CO}_{2}$-neutral energy supply the focus must, besides energy production, also be placed on carbon-neutral energy plants to provide ancillary services [1]. One of these services is Frequency Containment Reserve (FCR), which stabilises the grid frequency in the presence of fluctuations [2, 3]. Battery Energy Storage Systems (BESS) are well-suited for this service due their fast response characteristics. Lithium-Ion Battery Storages emit very low amounts of $\mathrm{CO}_{2}$ during operation and can therefore contribute to a carbon-reduced energy system [4]. Thus, there is an increased scientific interest in BESS for FCR in recent years (see Sct. 2), as well as an increase in installed capacity of BESS in industrial use [5].

Due to increasing competition in the FCR market and the resulting decrease in compensation rates, the financial margins of stand-alone battery storage systems are decreasing $[6,7]$. New innovative ways must be found to secure a competitive position.

Within the project "HyReK 2.0" (German: HybridRegelKraftwerk, English: Hybrid Regulating Power

Email address: uli.schlachter@dlr.de (Uli Schlachter)
Station), a battery storage is combined with a Powerto-Heat $(\mathrm{PtH})$ module. This sector integration approach allows converting excess electrical energy into heat and transferring it e.g. to a district heating network. The advantage of this hybrid system is the possible consumption of electrical energy by the $\mathrm{PtH}$ module, even if the battery storage is fully charged. Negative FCR, meaning the consumption of excess electric power in times of overfrequency, can therefore be provided via the battery storage or the PtH module. Thus, the battery's SoC can be higher in hybrid systems than in stand-alone BESSs. This approach allows the usage of a battery storage with a smaller storage capacity without violating the requirements for FCR provision. Since the battery investment is the largest part of the investment costs [8-10], this combination with a $\mathrm{PtH}$ module can be economically beneficial [11].

In this paper we consider three different system variants for providing $1 \mathrm{MW}$ of FCR:

- BESS: A system consisting of a stand-alone battery system. It needs an energy reserve at the lower and at the upper end of its $\mathrm{SoC}$ in order to fulfil the requirements for FCR provision. Energy is sold or bought at the electric power exchange to keep the $\mathrm{SoC}$ in the valid range. 
Table 1: List of abbreviations

$\begin{array}{ll}\text { BESS } & \text { Battery Energy Storage System } \\ \text { DoF } & \text { Degrees of Freedom } \\ \text { EPEX } & \text { European Power Exchange } \\ \text { FCR } & \text { Frequency Containment Reserve } \\ \text { HyReK } & \text { Hybrid Regulating Power Station } \\ \text { IDC } & \text { Intraday Continuous (Market) } \\ \text { NPV } & \text { Net Present Value } \\ \text { PtH } & \text { Power to Heat } \\ \text { SoC } & \text { State of Charge }\end{array}$

- Hybrid system: A system consisting of a combination of a battery storage with a PtH module. It only needs an energy reserve for low SoCs, since the energy consumption can take place via the $\mathrm{PtH}$ module irrespective of the battery's SoC. When the battery is fully charged, there are two different variants:

- HyReK-PtH: This variant uses the PtH module for energy consumption due to FCR provision when the battery is fully charged. In case the SoC is below a critical level, energy is bought at the power exchange.

- HyReK-Sell: This variant sells energy on the power exchange, thus preventing the battery from being fully charged. It also buys energy at the power exchange, when the SoC is low. If the energy cannot be sold in time, the $\mathrm{PtH}$ module is still used as backup.

For each of the variants different configurations are investigated. Their difference is in the battery capacity and SoC setpoint. Our focus will be to determine the operating strategy and battery capacity where economic use is maximized. The different operating strategies stem from a different utilisation of the degrees of freedom (DoF) that are available for the provision of FCR. This will be explained in more detail in Section 3.2.

In this paper, we consider the German energy market conditions. This is reflected in the assumed values of fees and levies for energy consumption, but also in the market prices for electricity at the power exchange and FCR remuneration.

An overview of the used abbreviations is provided in Table 1 .

In the next section, we outline the state of research. Afterwards, FCR is introduced in Section 3. Our approach is outlined in Section 4 and the results are presented and discussed in Sections 5 and 6, respectively. Section 7 concludes the paper.

\section{Related Work}

Much research was already published on batteries providing FCR. For example, different business cases for batteries are compared in $[12,13]$. The result is that FCR provision generates the highest revenue for these power plants. In this section, we give an overview over aspects investigated in the literature on FCR provision with battery storages. For our purposes, the important aspects are battery sizing, the use of the $\mathrm{DoF}^{1}$, and sector integration via a $\mathrm{PtH}$ module.

Sector integration was examined in the form of $\mathrm{PtH}$ $[11,14,15]$ and emergency resistors [8, 10] for dissipating energy when the battery is fully charged. In both cases, this has economic benefits, e.g. due to a reduction in battery investment.

Battery sizing is concerned with the capacity-to-power ratio of the battery, i.e. how much battery capacity is necessary for the provision of $1 \mathrm{MW}$ of FCR. This aspect is complicated by the fact that the regulatory framework in Germany recently changed: The 30 minutes criterion was replaced by the 15 minutes criterion $^{2}$, which has a huge effect on the necessary battery capacity.

Most papers consider only one or two battery capacityto-power ratios $^{3}[15-22]$. Other papers consider up to 12 different battery capacities, but do not answer the question what the optimal capacity for a stand-alone system is $[8,10,11,14,23]$. In an evaluation with 16 different battery capacities and the 30 minutes criterion, the highest economic benefit was provided by a system with a capacity-to-power ratio of 1.6 [24].

A review by Hesse et al. [25] of implemented systems shows that batteries are built with a power-to-energy ratio of about 1. Most papers also use this ratio, but values from 0.62 to 3 can be found. The high values usually stem from the assumption that the former 30 minutes criterion applies.

The DoF describe the allowed range of operation for the provision of FCR. They will be explained in detail in Sct. 3.2. Many authors conclude that the DoF can have a large effect on the operation of the battery storage. An overview of the effects of the individual DoF is given in various papers [16-19]. In these papers, the goal is mostly to minimise battery ageing.

Many authors use the DoF with an SoC setpoint. The goal is to operate the battery's SoC close to this setpoint by using the DoF, thus avoiding market costs. Fixed goals of $50 \%[20,21]$ or $60 \%$ [26] were investigated. When a PtH plant is considered, the DoF are most often used for maximal consumption, i.e., a setpoint of $100 \%$ is used $[8,11,15]$. An optimisation approach based on linear programming is taken in [18]. This leads to a perfect foresight into the future for the use of the DoF. The strategy uses the DoF to buy less and sell more energy, improving the financial margin by $3.5 \%$ when compared to without the use of the DoF. Thus, this is also a maximal consumption

\footnotetext{
${ }^{1}$ The DoF allow some variations in the operating strategy for FCR. They will be explained in detail in Section 3.2.

${ }^{2}$ See Sct. 3.4 for an explanation of the 15 minutes criterion.

${ }^{3}$ Often, more variations for other parameters are examined, e.g. the trade size on the market.
} 
strategy. Seven different SoC setpoints were considered in [16]. Values slightly above $50 \%$ yielded the highest economic benefits. In [23] a setpoint of $50 \%$ was compared with a setpoint depending on the market price. This price signal decreased the cost of market interactions. Fleer and Stenzel [19] used two SoC limits, between which minimal FCR is provided. Beyond these SoCs, the DoF are used to bring the battery's SoC back between the two limits. Three different values for the two boundaries were compared based on the amount of energy traded on the market. Since the least energy is traded on the market when the two limits are the same, a single setpoint seems to be sufficient.

Only few papers consider fees and levies, which amount to a large part of the energy consumption costs $[11,16]$.

In the present paper we aim to conduct an economic investigation of battery storage and hybrid systems with sector integration for the provision of FCR. We will identify the optimal battery capacity and SoC setpoint for the use of the DoF. In contrast to existing research, a large number of different parameter combinations is evaluated - 2048 combinations. This allows to identify the best system combination. Also, the relevant taxes and levies for the German market are considered, while a lot of existing research neglects them as a simplification. Since taxes and charges have a major influence on the profitability, their consideration leads to more realistic results.

\section{Frequency Containment Reserve}

In this section, an overview over the relevant regulations is provided $[2,3]$.

The nominal frequency of the European electricity grid is $50 \mathrm{~Hz}$. The actual frequency can deviate from this nominal value due to imbalances between production and consumption of energy. If more energy is produced than consumed, the rotating masses in conventional power plants gain kinetic energy and rotate faster than $50 \mathrm{~Hz}$. In the opposite case the grid frequency drops below $50 \mathrm{~Hz}$. The purpose of the FCR is to quickly react to these frequency deviations and to stop the increase or decrease. Therefore plants providing FCR have to consume or provide electrical energy to stabilise the grid frequency.

\subsection{Operation of Frequency Containment Reserve}

The deviation of the nominal grid frequency $\Delta f=$ $50 \mathrm{~Hz}-f$ is the main parameter for the provision of FCR power. At frequency deviations of $\pm 200 \mathrm{mHz}, \mathrm{FCR}$ power plants provide or consume their full marketed power $P$. Smaller deviations result in a proportional decrease of nominal FCR power $P_{\text {nom }}$, as indicated by the black line in Figure 1.

$$
P_{\text {nom }}(\Delta f)=P \cdot \begin{cases}1 & \text { if } \Delta f \geq 200 \mathrm{mHz} \\ \frac{\Delta f}{200 \mathrm{mHz}} & \text { if }|\Delta f|<200 \mathrm{mHz} \\ -1 & \text { if } \Delta f \leq-200 \mathrm{mHz}\end{cases}
$$

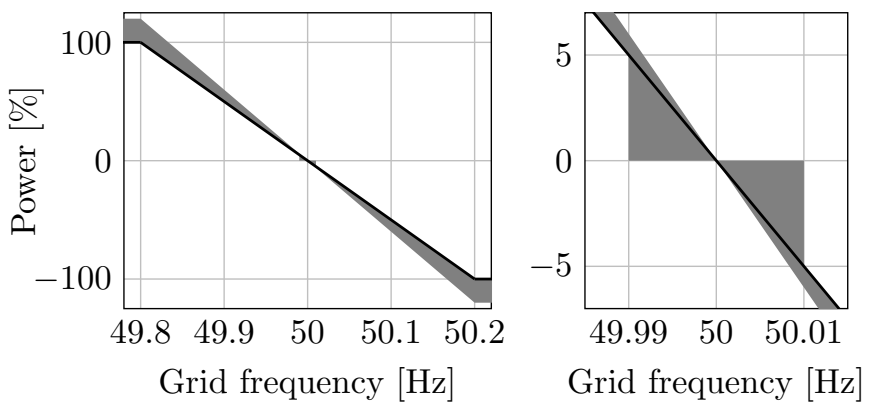

Figure 1: Power-frequency characteristic for providing FCR. Power limits for deadband and overfulfillment are indicated by the grey area. The left image shows the full frequency range while the right image emphasises the deadband.

\subsection{Degrees of Freedom (DoF)}

In Germany, there are several DoF for the provision of FCR. Power plants may use them in order to optimise their operation. In case of battery storages these DoF can be used to manage the SoC [27]. In this paper we mainly consider the DoF overfulfillment and deadband for driving the battery's SoC close to the setpoint. Additionally, plants can make transactions on the power exchange. This degree of freedom is used for keeping the $\mathrm{SoC}$ in the valid range for FCR provision.

Overfulfillment allows an energy output of up to $120 \%$ compared to the proportionally determined power. For small deviations of up to $\pm 10 \mathrm{mHz}$, the so-called deadband, power plants may ignore the deviation, providing no FCR power. These two DoF shall be used optimally by an operating strategy. Figure 1 visualises the influence of deadband and overfulfillment.

The actual upper and lower limits ${ }^{4}$ on the power output of an FCR providing plant are derived from these DoF. The upper limit is given by overfulfillment of up to $120 \%$ :

$$
P_{\max }(\Delta f)=1.2 P_{\text {nom }}(\Delta f)
$$

The lower limit follows the nominal FCR power, except inside the deadband, where no power needs to be provided:

$$
P_{\text {min }}(\Delta f)= \begin{cases}0 & \text { if }|\Delta f| \leq 10 \mathrm{mHz} \\ P_{\text {nom }}(\Delta f) & \text { if }|\Delta f|>10 \mathrm{mHz}\end{cases}
$$

The actual power output must be a value between $P_{\min }(\Delta f)$ and $P_{\max }(\Delta f)$.

In this paper, we want to answer the question how to make best use of the DoF.

\subsection{Charging/Discharging via Market Transactions}

Battery storages have a limited capacity. To ensure that FCR is continuously provided, balancing transactions are

\footnotetext{
${ }^{4}$ The upper limit is only larger with respect to its absolute value, i.e. $\left|P_{\max }(\Delta f)\right| \geq\left|P_{\min }(\Delta f)\right|$ holds and $P_{\max }(\Delta f) \geq P_{\min }(\Delta f)$ does not always hold.
} 


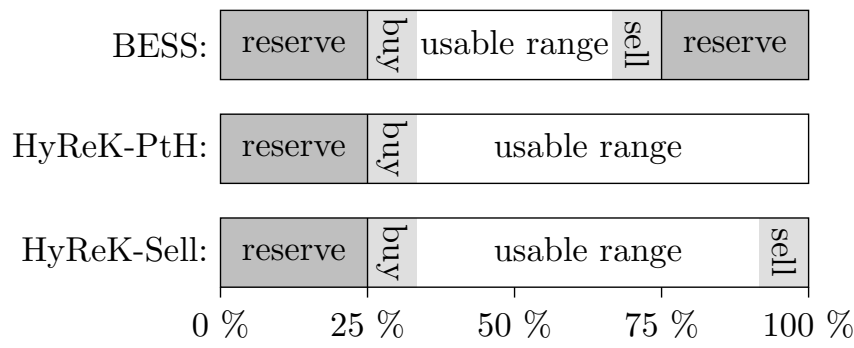

Figure 2: Visualisation of the different charge levels for a battery with $1 \mathrm{MWh}$ of capacity providing $1 \mathrm{MW}$ of FCR. The SoC is shown on the x-axis. The energy reserves for the 15 minutes criterion are indicated in dark grey. The ranges where market interactions become necessary are indicated in light grey.

necessary. This can be done via the intraday continuous (IDC) market of the European Power Exchange (EPEX SPOT) SE, because it allows making transactions on short notice. In Germany, each quarter hour is a time slice and transactions can be made up to five minutes before physical delivery ${ }^{5}$.

For example, when the battery's SoC enters the buy range indicated in Figure 2, energy is bought on the market. This should keep the SoC inside the usable range. This energy is provided as a constant power over 15 minutes. This extra power is taken into account for the FCR provision during this time.

\subsection{Energy Reserves for the "15 Minutes Criterion"}

FCR providing power plants have to fulfil the $15 \mathrm{~min}$ utes criterion: In normal operation, they have to be able to provide or consume the full FCR power for 15 minutes. A BESS must reserve some capacity for this criterion. In a system providing $1 \mathrm{MW}$ of FCR, 15 minutes of full power are equivalent ${ }^{6}$ to a reserve of $250 \mathrm{kWh}$. Because of the symmetry requirement, a total of $500 \mathrm{kWh}$ needs to be reserved: $250 \mathrm{kWh}$ of energy must always be available for provision and another $250 \mathrm{kWh}$ need to be consumable by the system. The upper part of Figure 2 illustrates the different energy ranges. Thus, this criterion limits the usable battery capacity range for FCR. In Germany, the 15 minutes criterion only recently replaced the 30 minutes criterion.

In case of the hybrid systems only the capacity for providing the full marketed power for 15 minutes must be reserved, since energy consumption is always possible via the $\mathrm{PtH}$ module. This is illustrated in the lower part of Figure 2.

To uphold the 15 minutes criterion, energy has to be sold or bought before this criterion is violated, as described in Section 3.3. In the worst case, a battery storage reaches a too high or too low SoC just after gate closure and has

\footnotetext{
${ }^{5}$ Only inside a control area. Across German control areas, transactions can be made up to 30 minutes before delivery. For crossborder trade, the gate closure time is 60 minutes.

${ }^{6}$ This explanation ignores energy conversion losses.
}

to wait for the next trading period. This can take up to 5 minutes for the gate closure time plus 15 minutes for the trading period, for a total of up to 20 minutes of so-called lag. According to regulations [3], one may assume a frequency deviation of $50 \mathrm{mHz}$ for this time period. When providing $1 \mathrm{MW}$ of FCR, this means that another $50 \mathrm{mHz} / 200 \mathrm{mHz} \cdot 1 \mathrm{MW} \cdot 20 \mathrm{~min}=1 / 4 \cdot 1 \mathrm{MW} \cdot 1 / 3 \mathrm{~h}=$ $1 / 12 \mathrm{MWh}$ of energy reserves are necessary to bridge the time until market transactions become active.

Besides the illustration of the energy reserves for the 15 minutes criterion, Figure 2 also shows how the usable range of BESS and HyReK-Sell is shrunk by the market lag (light grey area). This figure illustrates how the usable range of battery SoCs is larger for the hybrid systems than for a BESS. HyReK-PtH has the largest usable range, because it does not use an energy reserve for dealing with market lag when selling (indicated as a hatched area in the figure).

\subsection{FCR Remuneration}

The provision of FCR is remunerated by the power price, meaning that the power provided is remunerated irrespective of the electric energy consumed or provided. In July 2019, the time slices for the provision of FCR changed from one week to one day. Each participating power plant has to compete for a spot in the FCR market by placing a bid. The FCR offers are ranked according to their power price in the merit order and the lowest bids will be accepted until the required FCR power is secured. In July 2019 the remuneration scheme changed from pay-as-bid, meaning that offers are remunerated with the price that was bidden by them, to a uniform pricing scheme, meaning that offers are remunerated with the power price of the most expensive bid of an accepted offer [28]. In this paper, we use the former FCR market conditions.

\section{Method}

\subsection{Simulation Approach}

Our results stem from a simulation of FCR. For this, we simulate the provision of $1 \mathrm{MW}$ based on frequency data with a temporal resolution of 1 second and an accuracy of $1 \mathrm{mHz}$. The simulation is time discrete. At each time step, an operating strategy decides how to use the DoF and a new simulation state is computed. The state of the simulation includes the battery's SoC and the list of pending and active market transactions. Additionally, the cumulative energy flows are calculated for later analysis.

The battery storage SoC is kept within the valid boundaries via the power exchange. The simulation uses the average price per quarter hour of the IDC market. In all considered variants, energy is bought at low SoCs, and in some, energy is sold at high SoCs. The market interactions consider the rules for FCR: All trades are for $250 \mathrm{~kW}$, which is $25 \%$ of the prequalified power. Since a time slice lasts 15 minutes, this means that $250 \mathrm{~kW} \cdot 15 \mathrm{~min}=$ 
Table 2: Overview over the different simulations

\begin{tabular}{l|l} 
Parameter & Values \\
\hline system variants & BESS, HyReK-PtH, HyReK-Sell \\
battery capacity & $20 \mathrm{kWh}$ increments \\
setpoint & $3 \%$ increments \\
use of DoF & setpoint / ignored \\
scenario & with / without sell revenue
\end{tabular}

$62.5 \mathrm{kWh}$ of energy ${ }^{7}$ is traded in each transaction. Transactions are scheduled when there is less than 1/12 MWh of energy available in the battery before the 15 minutes criterion is violated. This number was calculated in Section 3.4 based on the regulations for FCR.

The simulation was repeated with different combinations of the parameters battery capacity and SoC setpoint for the three system variants. In total, 2048 different simulations were run.

Table 2 summarises the different simulations.

Battery ranges. The usable battery ranges for the three considered variants were calculated based on the criteria stated in Section 3.4 (see Figure 2):

- BESS: This variant needs energy reserves at the upper and lower end of battery charge levels. Thus, a BESS needs $2 \cdot(250 \mathrm{kWh}+1 / 12 \mathrm{MWh}) \approx 667 \mathrm{kWh}$ of energy reserves for the 15 minutes criterion and to deal with lag.

- Hybrid system: In a hybrid system, the PtH module can always consume energy. Thus, no energy reserves for consuming energy are necessary and only reserves for the provision of energy remain.

- HyReK-PtH: For HyReK-PtH this leads to a reserve of about $333 \mathrm{kWh}$.

- HyReK-Sell: For HyReK-Sell, we additionally have to consider the charge level above which energy is sold. We use the market lag value of $1 / 12 \mathrm{MWh}$. Energy reserves of $250 \mathrm{kWh}+2$. $1 / 12 \mathrm{MWh} \approx 417 \mathrm{kWh}$ are necessary.

Based on this, the minimum battery capacity was chosen to be $660 \mathrm{kWh}, 320 \mathrm{kWh}$, and $400 \mathrm{kWh}$, respectively. These numbers are deliberately chosen below the minimum capacity to check for an optimum close to the limit.

The battery capacity is incremented in $20 \mathrm{kWh}$ steps to construct the tested range of capacities, until $600 \mathrm{kWh}$ of capacity were added to the minimum capacity. The upper bound was chosen based on literature values and a pre-test. This means that 31 different battery capacities were tested

\footnotetext{
${ }^{7}$ This amount is less than the minimal possible transaction size of $100 \mathrm{~kW}$ on the IDC [29]. This complication is ignored and instead it is assumed that real systems provide more than $1 \mathrm{MW}$ of FCR and thus need larger market transactions. Our results can be scaled accordingly.
}

and the maximum capacity was $1260 \mathrm{kWh}, 920 \mathrm{kWh}$, and $1000 \mathrm{kWh}$ for BESS, HyReK-PtH and HyReK-Sell, respectively.

Operating strategies. The different operating strategies stem from a different utilisation of the DoF. Each operating strategy has an SoC setpoint. The available DoF are used to drive the battery's charge level towards the setpoint. For example, if energy has to be consumed while the SoC of the battery is already above the setpoint, only the minimal work allowed is provided. If however the battery SoC is below the setpoint and energy has to be consumed, the simulated plant provides the maximal amount of work that is allowed.

The possible values for the SoC setpoint are tested in $3 \%$ increments. For the BESS, values between $20 \%$ and $80 \%$ were tested. For HyReK, the range $20 \%$ to $101 \%$ was used. These numbers cover the usable range for all considered battery capacities.

Additionally, we examine the case of not using any DoF at all, but instead providing FCR purely proportional to the frequency deviation. This highlights the possible benefit due to the strategic utilisation of the DoF.

Scenarios. The simulation results were evaluated in different scenarios: With sell revenue and without sell revenue. In the scenario without sell revenue, selling energy on the power exchange does not generate revenue. This allows some insight into the actual operation of the plant and the use of 'free' energy from FCR provision. This is in contrast to with sell revenue in which the market interactions generate profit. HyReK-PtH does not sell energy, so its result are the same in both scenarios.

\subsection{Simulation Parameters}

In the simulation, data from July 2018 until the end of June 2019 were used. The simulated period was selected based on multiple considerations. It should cover at least one year, so that seasonal effects in grid frequency as well as electricity prices are included and because this simplifies the following economic evaluation. At the time of writing, the newest full year for which grid frequency data was available was 2018. However, at the beginning of 2018, there was a large grid time deviation which caused an unusual frequency profile (see e.g. [30]). Since this continuous deviation causes an asymmetric load on FCR providers, the experimental results would not be representative of usual power grid behaviour. Additionally, as described in Section 3.5, changes were made regarding the remuneration structure and time slices of FCR in July 2019. For this study, we want to consider only one set of regulations and therefore chose the former market structure.

We assume constant efficiencies for the system components. The battery has an efficiency of $95 \%$ and the $\mathrm{PtH}$ module of $99 \%$ (see e.g. [11]). This means that when the battery is charged with $1 \mathrm{MWh}$, its stored energy increases by $0.95 \mathrm{MWh}$. To return the SoC to its initial value, about 
Table 3: Economic simulation parameters

\begin{tabular}{l|l} 
Parameter & Value \\
\hline Investment cost battery & $600 € / \mathrm{kWh}$ \\
Investment cost $1 \mathrm{MW} \mathrm{PtH}$ & $25,000 €$ \\
Fixed investment cost & $150,000 €$ \\
FCR revenue & $100,393 € /$ Year \\
Maintenance cost & $2 \%$ of total invest. p.a. \\
Discount rate & $5 \%$ \\
System lifetime & 15 years \\
Consumption fees BESS & $9.95 € / \mathrm{MWh}$ \\
Consumption fees PtH & $157.78 € / \mathrm{MWh}$ \\
Revenue for district heating & $10 € / \mathrm{MWh}$
\end{tabular}

0.9 MWh need to be discharged. Therefore, the battery has a round-trip efficiency of $90.25 \%$.

The economic simulation parameters are shown in Table 3. The FCR revenue is the sum of the actual average prices in the considered time period. No downtime for maintenance was included. The value of the charges and levies for electricity consumption of $\mathrm{PtH}$ and BESS derives from the levies on electricity purchases applicable in Germany in $2019^{8}$. Since the battery storage feeds the stored electric energy back into the power grid, it is exempt from certain charges. The $\mathrm{PtH}$ module is burdened with all charges, which explains the different values for fees and levies.

Fixed levies, which are incurred regardless of the amount of electricity purchased, are neglected. Fixed investment costs include costs for the power electronics as well as necessary infrastructure. The battery capacity investment costs of $600 € / \mathrm{kWh}$ includes not only the individual cells, but e.g. also necessary housing. This value is a rough estimate which will be further discussed in Section 6.5.

\subsection{Net Present Value Indicator}

The simulation results for one year were used to calculate a net present value for the system. This approach computes a single indicator based on the cash flow in each time period. The investment cost was assumed to occur at the beginning. Afterwards, the cash flow given by the simulation was repeated 15 times.

$$
\mathrm{NPV}=\sum_{t=0}^{T} \frac{C_{t}}{(1+r)^{t}}
$$

$T$ is the system lifetime in years, $C_{t}$ is the cash flow in year $t$, and $r$ is the discount rate.

\section{Results}

In this section, we present the simulation results. First the results for the scenario with sell revenue are given. The

\footnotetext{
${ }^{8}$ Relevant taxes and charges are electricity tax, value added tax, grid fees, concession levy, renewable energy charge, combined heat and power charge, offshore grid charge, $\S 19$ charge, and curtailable load charge.
}

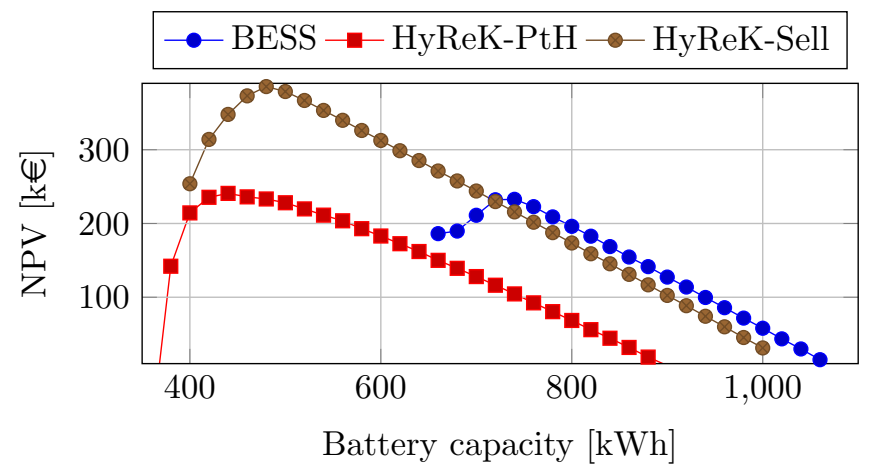

Figure 3: NPV for all three system variants when the DoF are not used.

results for the simulations in which FCR is provided proportionally to the frequency deviation, meaning that the DoF are ignored, are shown in Section 5.1. Afterwards, the results for operating strategies with an SoC setpoint are presented in Section 5.2. Second the results for the scenario without sell revenue are presented in Section 5.3 and Section 5.4 without and with the use of the DoF, respectively.

\subsection{Results Without SoC Setpoint With Sell Revenue}

Figure 3 shows the results for the configurations that do not use an SoC setpoint. Instead, these configurations always provide FCR proportional to the frequency deviation, without using any DoF. In this case the optimal battery capacity is $740 \mathrm{kWh}$ for the BESS with an NPV of $233 \mathrm{k} €$. HyReK-PtH has the best NPV for a capacity of $440 \mathrm{kWh}$ with $241 \mathrm{k} €$ and the HyReK-Sell achieves an NPV of $386 \mathrm{k} €$ with $480 \mathrm{kWh}$ of battery capacity. These results already highlight that the hybrid system can provide FCR with smaller battery capacities. Additionally, this has financial benefits, as can be seen from the higher NPV of HyReK-Sell.

The NPV changes differently above the optimal battery capacity compared to below it. Larger battery capacities lead to a near linear decrease of the NPV with a slope of about $-700 € / \mathrm{kWh}$. Too small battery capacities produce a higher increase in operating costs (energy fees and trading costs) than the lower investment costs can compensate, resulting in a fast decrease of NPV. The curves do not appear linear and computing slopes is not sensible for this case.

\subsection{Results With SoC Setpoint With Sell Revenue}

We present the NPVs, battery capacities and setpoints of the different variants and configurations targeting an SoC setpoint. For each system variant, the results are shown in one plot.

BESS. The results for the BESS are shown at the top of Figure 4. The highest NPV has a value of $284 \mathrm{k} €$ for a battery capacity of $740 \mathrm{kWh}$ and an SoC setpoint of $71 \%$. 
BESS:

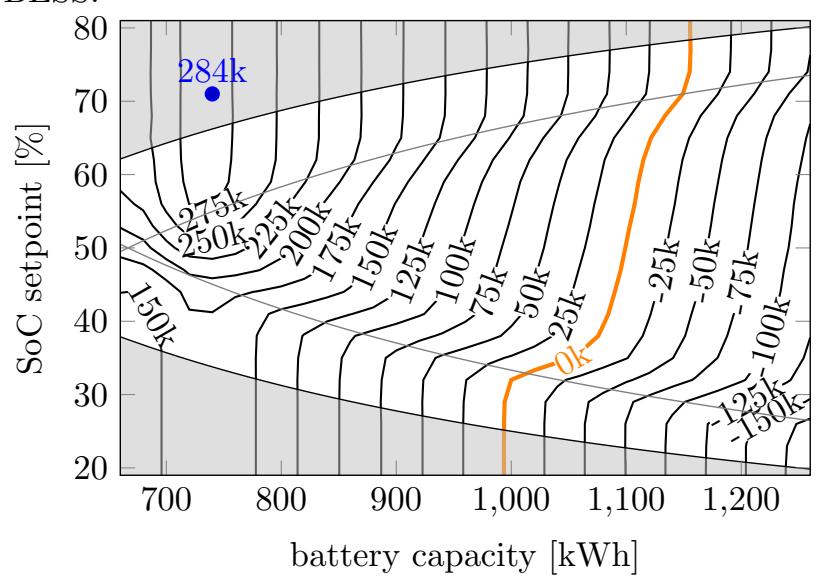

HyReK-PtH:

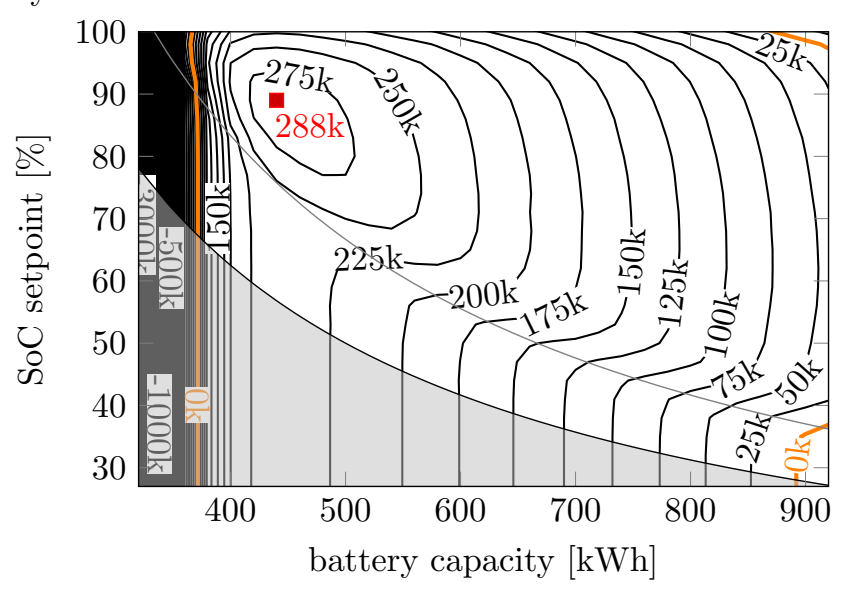

HyReK-Sell:

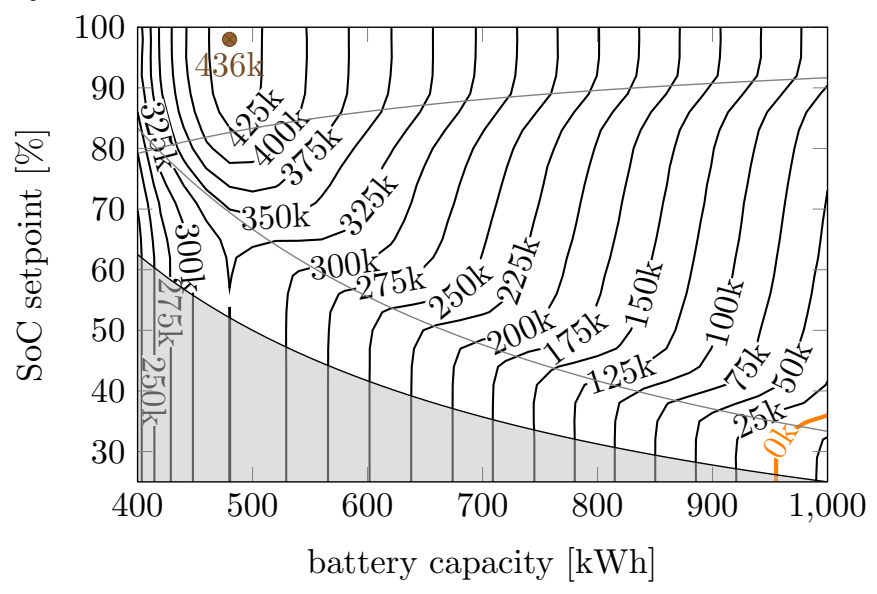

Figure 4: NPV for BESS, HyReK-PtH, and HyReK-Sell, respectively, as contour plots. The best NPV is marked. The grey areas are SoCs were the 15 minutes criterion is violated. The grey lines that are parallel to the borders of the grey area or to the top of the graph indicate the border beyond which market transactions are activated.
As can be seen in the figure, the $\mathrm{SoC}$ setpoint is inside the energy reserves for the 15 minutes criterion. This means the DoF are used for maximal energy consumption. Small changes to the SoC setpoint do not deviate from the best result much, but changes to the battery capacity cause a large decline in NPV. For example, a battery with a capacity of $1000 \mathrm{kWh}$ is only $260 \mathrm{kWh}$ above the optimal battery capacity, but the best NPV is reduced by more than half.

In this plot, one can see the importance of setpoints inside the range for market transactions. The grey lines indicate the borders where market transactions are activated. Between these two lines, the NPV changes relatively smoothly when modifying the SoC setpoint, but near the lines the NPV changes more abruptly. Beyond these borders, the lines continue vertically. This is also true for the area covered in grey, representing areas in which the 15 minutes criterion is violated.

HyReK-PtH. The hybrid system HyReK-PtH is considered in the middle of Figure 4. This is the variant that does not sell energy when the battery's SoC is near the upper border, because electric energy is then consumed by the PtH module. The largest NPV occurs for a battery with $440 \mathrm{kWh}$, which is $107 \mathrm{kWh}$ above the minimal capacity, and an SoC setpoint of $89 \%$. It amounts to $288 \mathrm{k} €$. Lower battery capacities lead to a quickly decreasing NPV, as is evident by the many close contour lines on the left side of the image. For larger battery capacities, the slope is relatively low.

HyReK-Sell. The hybrid system HyReK-Sell is considered at the bottom of Figure 4. When the hybrid system can sell energy at high charging states, it avoids the high fees for using the PtH module. Thus, smaller battery capacities and higher SoCs become profitable for HyReK-Sell. The best NPV for this system is $436 \mathrm{k} €$ and it occurs with a battery capacity of $480 \mathrm{kWh}$ and an SoC setpoint of $98 \%$. The optimal battery capacity is therefore $63 \mathrm{kWh}$ above the minimal value. Again, the NPV decreases faster at lower battery capacities than at higher ones.

\subsection{Result Without SoC Setpoint Without Sell Revenue}

Figure 5 shows the results of the simulation in which FCR is provided proportionally to the grid frequency deviation without using the DoF. Here, energy is given away for free at high SoCs and therefore does not generate revenue.

The BESS performs best with $760 \mathrm{kWh}$ of battery capacity and has an NPV of $172 \mathrm{k} €$. The best NPV for HyReK-Sell is $320 \mathrm{k} €$ which occurs with $520 \mathrm{kWh}$ of battery capacity. Since HyReK-PtH does not sell energy, the results are still the same as in Figure 3. Its best NPV occurs with a capacity of $440 \mathrm{kWh}$ and is $241 \mathrm{k} €$.

For the BESS, small battery capacities become highly unprofitable, as can be seen on the left side of Figure 5 . The NPV of the HyReK-Sell also decreases significantly 


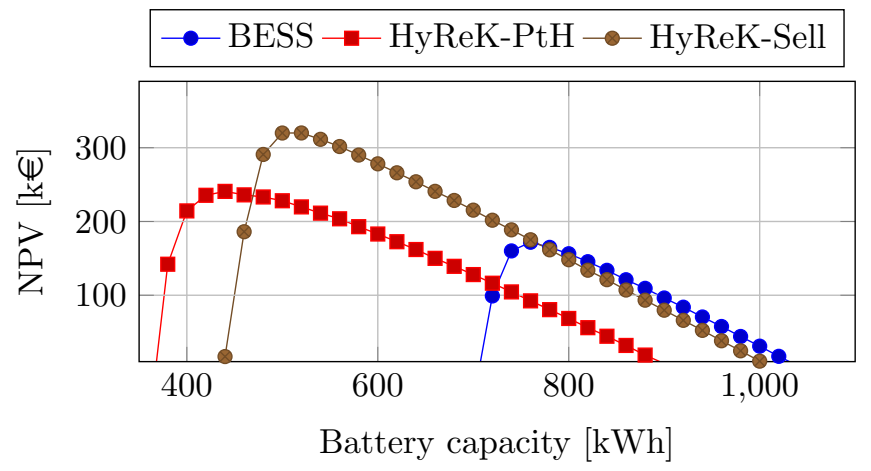

Figure 5: NPV for all three system variants when the DoF are not used and no revenue is generated by selling energy. The results for HyReK-PtH are unmodified compared to Figure 3, because this variant does not sell energy.

more than before as the battery capacity decreases. The cost for buying the energy now influences the NPV more negatively, since selling energy does not generate any revenue to counterweight the cost.

\subsection{Result With SoC Setpoint Without Sell Revenue}

In the following the results of BESS and HyReK-Sell are given in the scenario without sell revenue in which DoF are used. Since this case does not make any difference for HyReK-PtH the results of this variant are not shown here.

BESS. The highest NPV for the BESS occurs with $760 \mathrm{kWh}$ of battery capacity and an SoC setpoint of $53 \%$. It amounts to $194 \mathrm{k} €$ and is shown at the top of Figure 6. The optimal battery capacity is $93 \mathrm{kWh}$ above the minimal capacity. It can be seen that smaller battery sizes lead to a strong reduction of the NPV in this case. The NPV decrease at larger than optimal battery sizes is moderate.

HyReK-Sell. The hybrid system HyReK-Sell is considered at the bottom of Figure 6. Even though this system cannot generate revenue through selling electricity at the energy exchange, it can give away electric energy for free, avoiding high fees and taxes for using the $\mathrm{PtH}$ module. It has an optimal configuration of $500 \mathrm{kWh}$ of battery capacity, which is $83 \mathrm{kWh}$ above the minimal value, and an SoC setpoint of $80 \%$ with an NPV of $344 \mathrm{k} €$.

\section{Discussion}

The results obtained in the simulation of the three variants BESS, HyReK-PtH and HyReK-Sell are discussed below. Both optimal and non-optimal system configurations are considered. Non-optimal battery sizes and nonoptimal SoC setpoints are discussed separately. Furthermore, violations of the 15 minutes criterion and the general limits of the study are discussed.

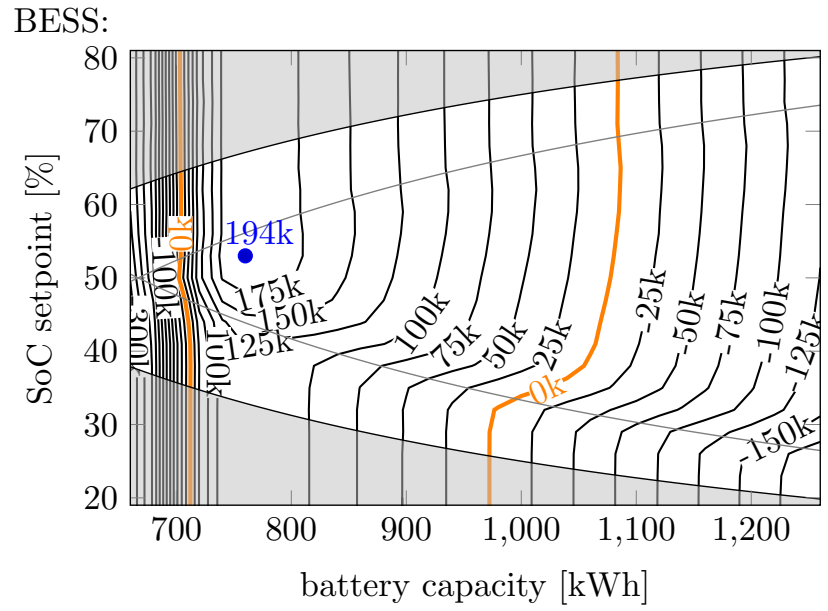

HyReK-Sell:

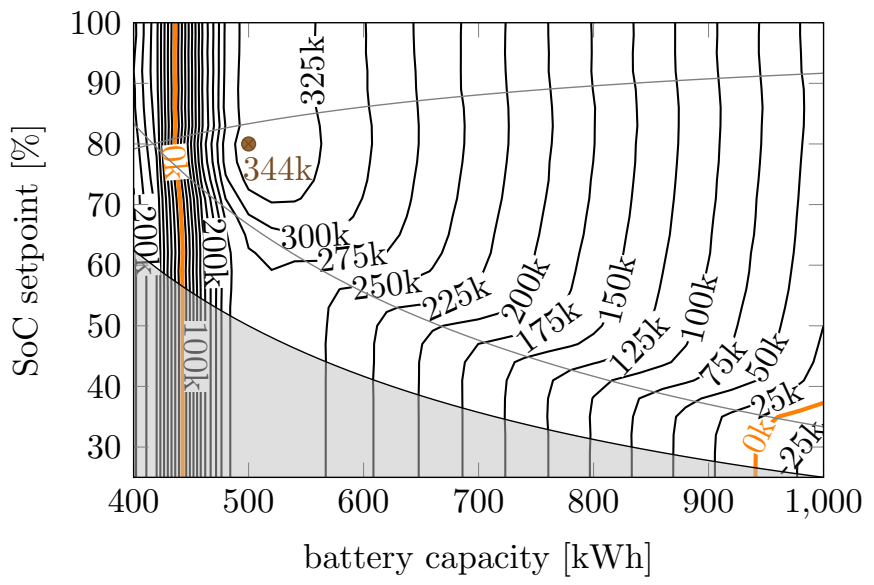

Figure 6: NPV for BESS and HyReK-Sell without sell revenue, cf. Figure 4. HyReK-PtH is not included because its results did not change.

\subsection{Optimal System Configurations}

Comparison of Variants. For each considered variant, there are battery capacities and SoC setpoints resulting in the highest NPV. These optimal system configurations and their corresponding NPVs are summarised in Table 4. To be able to better compare the best NPVs, Figure 7 illustrates this data from Table 4.

It can be clearly seen that the hybrid variants always show a higher profitability than the BESS, regardless of the scenario under consideration. The same relationship can be observed between HyReK-PtH and HyReK-Sell, since HyReK-Sell generates the higher NPV independent of the scenario.

This shows that HyReK-Sell has a large advantage over the other two variants, especially when selling energy generates revenue. If energy is given away for free, the best NPV for HyReK-PtH does not change, because this variant does not sell energy. Since the NPV of the other two system variants decreases in the scenario without sell revenue, HyReK-PtH now has a larger advantage over the BESS. However, HyReK-Sell still generates a higher NPV 
Table 4: Summary of best system configurations in each scenario.

With sell revenue

\begin{tabular}{clccc} 
DoF & Variant & Capacity & Setpoint & NPV \\
\hline setpoint & BESS & $740 \mathrm{kWh}$ & $71 \%$ & $284,197 €$ \\
setpoint & H.-PtH & $440 \mathrm{kWh}$ & $89 \%$ & $288,469 €$ \\
setpoint & H.-Sell & $480 \mathrm{kWh}$ & $98 \%$ & $435,605 €$ \\
\hline ignored & BESS & $740 \mathrm{kWh}$ & - & $232,768 €$ \\
ignored & H.-PtH & $440 \mathrm{kWh}$ & - & $240,812 €$ \\
ignored & H.-Sell & $480 \mathrm{kWh}$ & - & $385,612 €$ \\
\hline
\end{tabular}

Without sell revenue

\begin{tabular}{clccc} 
DoF & Variant & Capacity & Setpoint & NPV \\
\hline setpoint & BESS & $760 \mathrm{kWh}$ & $53 \%$ & $194,417 €$ \\
setpoint & H.-PtH & $440 \mathrm{kWh}$ & $89 \%$ & $288,469 €$ \\
setpoint & H.-Sell & $500 \mathrm{kWh}$ & $80 \%$ & $344,445 €$ \\
\hline ignored & BESS & $760 \mathrm{kWh}$ & - & $171,866 €$ \\
ignored & H.-PtH & $440 \mathrm{kWh}$ & - & $240,812 €$ \\
ignored & H.-Sell & $520 \mathrm{kWh}$ & - & $320,014 €$ \\
\hline
\end{tabular}

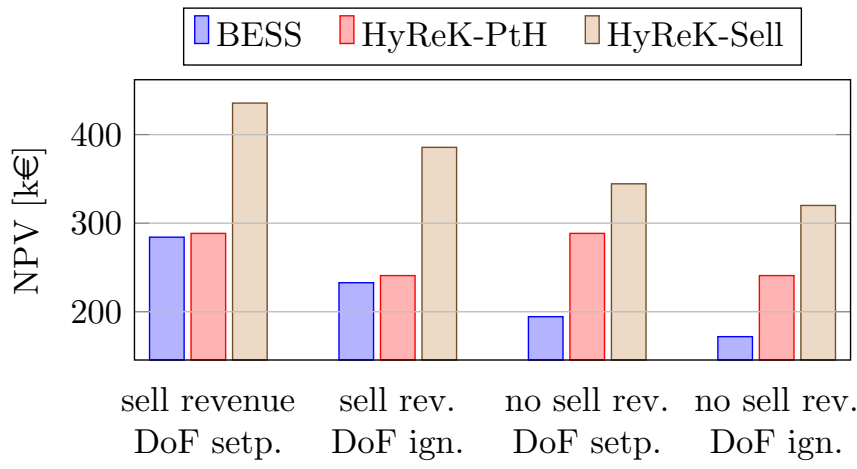

Figure 7: Illustration of best NPVs in each considered scenario.

than HyReK-PtH, because giving energy away for free is still cheaper than using the $\mathrm{PtH}$ module with its large fees and taxes. Another insight is the positive effect of the DoF on the results: Comparing the scenario with sell revenue with and without the usage of the DoF, the NPV increases by about $50 \mathrm{k} €$ in each system variant. When sell revenue is ignored, the improvement due to the DoF is still about $20 \mathrm{k} €$. Thus, even though the DoF seem relatively small, they have a noticeable effect.

Optimal Capacity. In all the considered scenarios, the optimal battery capacity for the two hybrid variants is about $40 \%$ smaller than the optimal battery capacity of the BESS. It was expected that the optimal capacity is smaller because of the smaller energy reserves for the hybrid variants, which result in a difference of $250 \mathrm{kWh}$ for HyReKSell and $333 \mathrm{kWh}$ for HyReK-PtH.

Also, between the scenarios, the optimal battery capacity of a system variant stays about the same. The greatest variations occur for HyReK-Sell. Its optimal battery capacity varies between $480 \mathrm{kWh}$, in the case with sell revenue, and $520 \mathrm{kWh}$, in the case without sell revenue and without the usage of DoF. For the variants BESS and HyReK-Sell the optimal battery capacities tend to be slightly larger in the scenarios without sell revenue. This might be due to higher benefits of storing electric energy in the storage since giving it away generates no profits anymore. In all but one cases, optimal capacities in the scenarios with setpoint equal the ones in the scenarios without setpoint. Exception here is HyReK-Sell in the scenario without sell revenue. Here the optimal capacity is higher in the case without SoC setpoint.

In the literature often high capacity to power ratios of at least $1 \mathrm{MWh}$ to $1 \mathrm{MW}$ are considered (e.g. [10, 11, 16, 19$22,26])$. However, our results clearly show that a lower ratio offers economic advantages both in the case of hybrid systems and in the case of BESS.

Optimal SoC Setpoint. For the SoC setpoint, one could intuitively expect an optimal value that is in the middle of the usable battery range, so that the SoC limits are far away when the setpoint is reached. However, this might only be true in situations in which the upper and lower SoC limits have about the same conditions. This can nicely be seen in the optimal setpoint of the BESS in the scenario without sell revenue. Here the limits for fulfilling the 15 minutes criterion as well as limits for buying electricity and giving it away for free are equal on both SoC ends. Additionally neither buying nor giving electricity away for free provides revenue in most situations, resulting in an optimal SoC setpoint of $53 \%$. Reasons why the setpoint is not in the exact middle might be the efficiency of the charging and discharging processes as well as the cost occurring for buying electricity in case the lower border is reached. In the scenario without sell revenue, unlike in the case of BESS, the two hybrid variants have a relatively high SoC setpoint. This could result, as in the case of BESS, from the efficiency losses during charging and discharging. Above all, however, one main factor may be the fact that the middle of the usable range of the hybrid systems is not equal to the middle of the overall battery capacity, like in case of BESS, due to the missing upper limit for the 15 minutes criterion. Thus, the middle of the usable range of the HyReK-PtH is an SoC of $87.84 \%$ and an SoC of $75.03 \%$ for HyReK-Sell.

As explained, the optimal battery capacities of the hybrid variants are significantly smaller than the optimal battery capacity of the BESS. Since all three variants provide the same amount of FCR, namely $1 \mathrm{MW}$, it seems to be economically reasonable, according to the results of the simulation, to keep a similar absolute amount of energy in the battery storage in the scenario without sell revenue. Due to the different $\mathrm{SoC}$ setpoint and different battery capacities, the three variants contain similar absolute amounts of energy in the battery storage in their optimal configuration. The BESS holds $402 \mathrm{kWh}$, HyReKPtH $392 \mathrm{kWh}$, and HyReK-Sell $400 \mathrm{kWh}$ of energy.

In the literature, the DoF are often used for maximal consumption (e.g. [8, 11, 15, 18]). Our results in the scenario with sell revenue confirm this as the optimal use. 


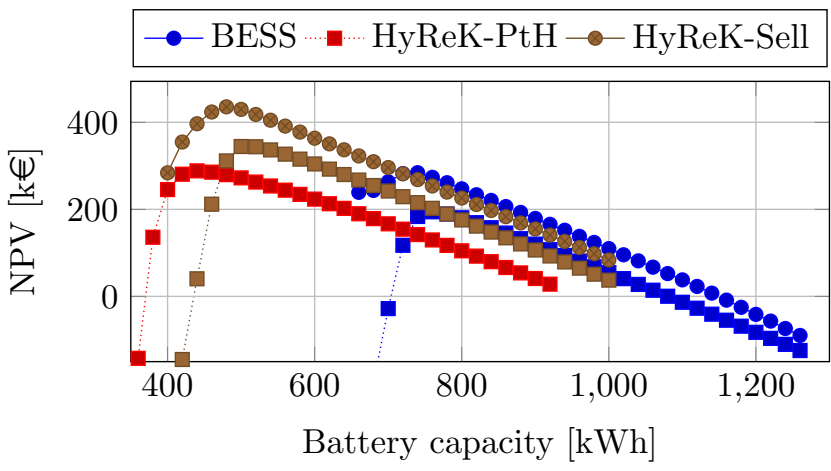

Figure 8: NPVs with varying battery capacities and fixed and optimal SoC setpoints. BESS has setpoint $71 \% / 53 \%$, HyReK-PtH has setpoint $89 \%$, HyReK-Sell has setpoint $98 \%$ / $80 \%$. The solid lines with round markers show scenarios with sell revenue and the dotted lines with square markers show scenarios without sell revenue.

However, for a hybrid system, it is better to avoid the high operational cost for the $\mathrm{PtH}$ module.

Sell Revenue. While for HyReK-PtH the situation does not change whether or not selling electricity generates revenue, the situation for BESS and HyReK-Sell changes drastically. In the scenario with sell revenue, both variants can consume energy at nearly no cost when providing FCR and then sell this energy on the market for a profit. This causes the optimal SoC setpoints to be at the upper end of the valid battery range in these cases. Here it must again be noted that the reserves for meeting the 15 minutes criterion are different at the upper limit for BESS and HyReK-Sell, since HyReK-Sell has no reserve at the upper SoC range, so that the absolute values of the SoC setpoints are not directly comparable. Nevertheless in both cases the SoC setpoint increases by $18 \%$ in the scenario with sell revenue compared to without sell revenue.

\subsection{Non-Optimal Battery Capacities}

The effect of non-optimal battery capacities is shown in Figure 8. This figure shows a subset of the data from Figures 4 and 6: The battery capacity is still varied, but the SoC setpoint is kept constant. The SoC setpoint from the optimal system configuration is used for each variant.

It can be seen that systems with too small or too large battery capacities always lead to decreasing NPVs.

Battery capacities that are larger than the optimal system configuration lead to a moderate, almost linear decline of the NPV of about $-700 € / \mathrm{kWh}$. The value of the NPV reduction corresponds to the additional battery investment cost as well as the additional maintenance cost which accrue every year for the additional battery capacity. One can conclude that battery capacities above the optimum hardly lead to any reduction of operating cost, but only cause higher investment and maintenance cost.

If the battery capacities are smaller than the optimal battery capacity, the NPV decreases significantly faster, especially in the scenarios without sell revenue. The main

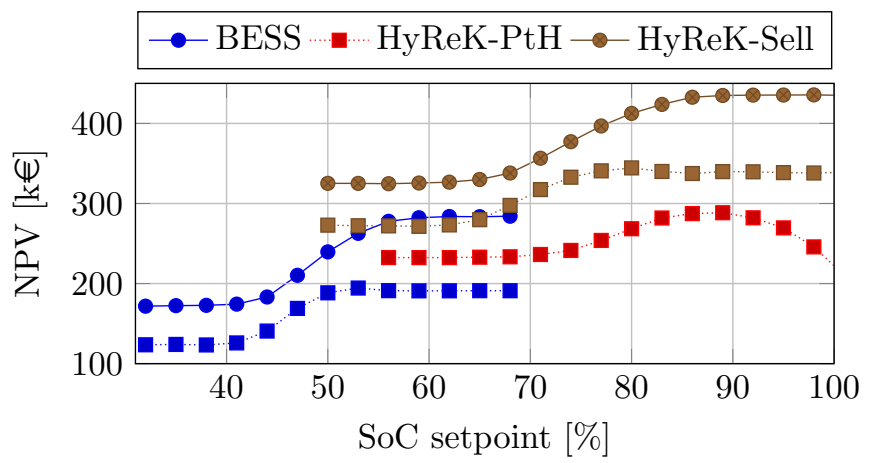

Figure 9: NPVs with varying SoC setpoints and fixed and optimal battery capacities. BESS has $740 \mathrm{kWh} / 760 \mathrm{kWh}$ capacity, HyReK$\mathrm{PtH}$ has $440 \mathrm{kWh}$ capacity, HyReK-Sell has $480 \mathrm{kWh} / 500 \mathrm{kWh}$ capacity. The solid lines with round markers show scenarios with sell revenue and the dotted lines with square markers show scenarios without sell revenue.

reason for this is that as battery capacities decrease, the usable range eventually becomes so small that the limits for buying and selling electricity meet or even overlap. In these cases the systems oscillate between buying and selling energy. In our simulation, preference was given to buying energy when the limits overlap. Thus, a lot of money is spent for buying energy, which is especially disadvantageous when no sell revenue is generated. With HyReK-PtH, high costs occur for the energy that is consumed by the PtH module. This leads to a steep gradient for these systems. When sell revenue is considered, these steep gradients in the NPV reduction disappear, but the trading cycles still occur.

\subsection{Non-Optimal SoC Setpoints}

Just like non-optimal battery capacities, non-optimal SoC setpoints also have a negative effect on the NPV. Figure 9 shows the course of the NPVs of the three system variants in the different scenarios, having this time fixed battery capacities and varying SoC setpoints.

In all but one variant, the NPV generally increases with higher SoC setpoints. The exception to this is HyReK$\mathrm{PtH}$ where a high setpoint results in more energy being consumed by the $\mathrm{PtH}$ module, which causes high costs. However, the NPV does not increase linearly with increasing SoC setpoints, but changes most significantly between the limits for buying and selling energy.

Around the limit for buying energy and the limit for selling energy, changes to the setpoint have almost no effect on the NPV. Only between the limits does the resulting NPV react to changes in the SoC setpoint.

Systems with an SoC setpoint close to the limit for buying energy need to recharge frequently, resulting in unnecessarily high costs. Systems that sell electric energy profitably at a high charge level have hardly any chances to use this source of income at such a low SoC setpoint, because they rarely reach a charge level that leads to a sale of electric energy. This can be seen when comparing a sys- 


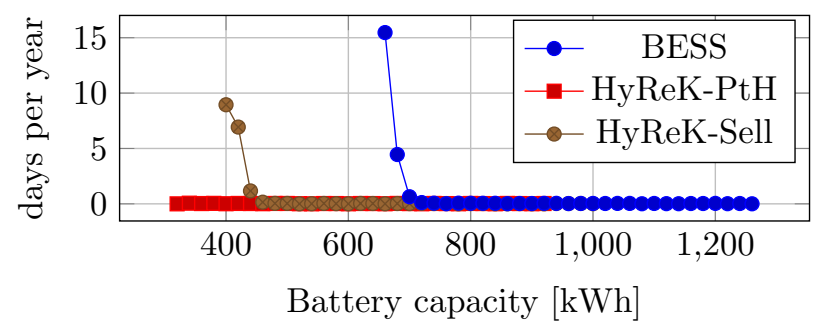

Figure 10: Violations of the 15 minutes criterion for system configurations without SoC setpoints.

tem in the with sell revenue and without sell revenue scenarios: The difference between the two scenarios increases with higher SoC setpoint. With low SoC setpoints, the advantage due to selling decreases.

The slower increase of the NPV in scenarios with sell revenue above the sell limit can be explained by the fact that the systems already sell a lot of energy at high SoC setpoints. Once the sell limit is exceeded, it is hardly possible to further increase the sold quantities and with that the generated revenue. The same applies to the scenarios without sell revenue, in which the discharging of the battery storage cannot be increased much further after crossing the upper limit. Here, however, the higher cost due to the increased utilization of the DoF for power consumption is more significant since the discharging does not generate revenue. This results in an earlier reduction of NPV increase and even slightly decreasing NPVs at high SoC setpoints.

Compared to the non-optimal battery capacities, the effects of non-optimal setpoints on the NPVs are smaller. While NPVs even become negative at non-optimal battery capacities (Figure 8), the largest NPV reduction due to non-optimal SoC setpoints is in the range of just over $100 \mathrm{k} €$ in the case of HyReK-Sell with sell revenue (Figure 9). While setpoints can be monitored and corrected during operation, the battery capacity is a critical investment decision with large effects.

\subsection{Violation of the "15 Minutes Criterion"}

Another aspect is the quality of FCR provision, namely how often the systems violate the 15 minutes criterion during operation. Figure 10 shows the proportion of time in which this criterion is violated by the systems without SoC setpoints. Results for the configurations with setpoints are similar.

It can clearly be seen that a lower battery capacity, and therefore a smaller usable range, lead to more frequent violations of the 15 minutes criterion. As already described, a too small battery capacity leads to an overlap of the selling and buying ranges. This means that there can be situations in which the system has to buy and sell energy at the same time, because both borders of the 15 minutes criterion are too close. In our simulation, preference was given to buying energy in this case. The effect of this decision is a less frequent violation of the 15 minutes criterion by HyReK-PtH compared to the other variants, because it only buys but never sells energy. Since it only has a range for buying energy, no overlap is possible.

However, all variants sometimes violate the 15 minutes criterion, even with large batteries. This can be explained by the calculation of the recharge limit assuming a frequency deviation of at most $50 \mathrm{mHz}$ (see Sct. 3.4). If larger frequency deviations occur, violations of the 15 minutes criterion are hardly avoidable.

Assuming that a violation of the 15 minutes criterion in less than 0.5 percent of the time, or 1.825 days per year, is considered to be a sufficiently high quality FCR provision (e.g. used in [24]), the different system variants require certain minimum capacities. Compared to the minimal viable battery capacities which were calculated in Section 3.4, the BESS needs at least $33 \mathrm{kWh}$ of usable battery capacity, resulting in a total battery capacity of $700 \mathrm{kWh}$. HyReK-Sell needs at least $23 \mathrm{kWh}$ of usable battery range, resulting in a minimal battery capacity of $440 \mathrm{kWh}$. A stand-alone $\mathrm{PtH}$ module can provide FCR without a battery ${ }^{9}$ by constantly buying energy and varying the consumption as required. For this reason, HyReK-PtH almost never violates the 15 minutes criterion, so that here the requirement for sufficient FCR quality is fulfilled regardless of the battery capacity.

Since the battery capacity was only examined in $20 \mathrm{kWh}$ increments, the required usable ranges for BESS and HyReK-Sell are not precise. Future work could deal with this in more detail.

\subsection{Limits of this Study \& Future Work}

We simulated one year of FCR operations. This year was selected to be representative (see Section 4.2). Although some tests for different time periods had similar results, future frequency profiles can be different and large deviations can occur again. This inevitably leads to uncertainties.

In addition, some simplifying assumptions were made when conducting the study. We assumed no downtime for maintenance or failures in the lifetime of 15 years and that all tenders on the FCR market are accepted. The district heating grid can always accept the energy consumed by the PtH module.

Market Interactions. We used a simplified model of market interactions. The assumption that electricity can be bought or sold five minutes before physical delivery is simplifying for two reasons. Firstly, the trading systems require some processing time. This processing needs to be finished at gate closure. Secondly, the liquidity of continuous intraday trading is relatively low. In 2018, the trading volume of the intraday market was about five times smaller than the volume on the day-ahead market [29]. Thus, it

\footnotetext{
${ }^{9}$ This is a technical possibility. Economically, the energy costs and taxes make this approach unattractive.
} 
can happen that, e.g., a buying bid cannot be matched with a selling bid in time. Also, the price that was bid could be too high/low so no matching bid exists. In addition, the gate closure time of five minutes before delivery only applies to plants within a control area, which limits the available market liquidity further. Making a successful trade whenever necessary is thus a very optimistic assumption in the simulation.

Battery Ageing. Battery ageing is neglected in this paper. In general, battery ageing models consist of two parts: Cyclic ageing and calendric ageing. While cyclic ageing depends on the charging and discharging cycles of the battery and therefore on the operation of the system, calendric ageing also occurs when the battery storage is not used [31]. In FCR provision, calendric ageing is the main factor for battery ageing [24]. Most research on battery ageing was done in the context of electric mobility, where clear cycles occur. In an FCR application, these models have limited applicability. Various effects play a role for both forms of ageing, which mostly cannot be considered individually and therefore further complicate the issue [32]. Due to the high uncertainty of this factor, it was neglected in the considerations.

Battery Investment Cost. Many different investment costs for lithium-ion battery storages are assumed in the literature, ranging from 100 to $900 € / \mathrm{kWh}$ (e.g. [19, 31, 33]; some cell chemistries cost up to $2200 € / \mathrm{kWh} \mathrm{[34]).}$ $600 € / \mathrm{kWh}$ was also used in $[11,20]$. The assumptions for battery cost made in this simulation is quite pessimistic. More optimistic assumptions with lower battery cost, based on the assumption that investment costs will further decrease in the future, lead to an increased NPV in the three variants. For BESS this increase will be stronger than for the hybrid variants, since lower battery investment cost plays a smaller role for the latter due to the lower battery capacity. In such a case, the BESS could achieve a higher NPV than the HyReK-PtH in the optimum configuration. The large difference between the NPVs of the BESS and HyReK-Sell would also be reduced, although the HyReK-Sell would probably still be the more profitable energy system for the foreseeable future. A sensitivity analysis regarding the influence of the investment cost of the battery storage on the NPV of the three system variants as well as on their optimal configuration would also be an interesting extension of the study conducted here.

\section{Conclusion}

In this paper, we investigated optimal system configurations for providing FCR with three different system variants: A classical BESS and a combination of a battery storage with a $\mathrm{PtH}$ module, for which two variants were considered: In the first one, the $\mathrm{PtH}$ module is used when the battery is fully charged, which was called HyReK-PtH.
The second one, HyReK-Sell, sells energy at high SoCs. This avoids the high taxes for energy consumption with the PtH module.

The optimal system configurations were sought for the three variants under different conditions. A distinction was made between the provision of FCR according to the deviation of the grid frequency and the provision of FCR while using the DoF to achieve a specific SoC setpoint. In addition, differences in the optimal system configuration of the variants BESS and HyReK-Sell were investigated for the case where electricity sales generate revenues and for the case where electric energy is given away for free at high SoC setpoints.

The results show that the best NPV generally occur for small battery capacities that only have a small range of allowed SoCs. After subtracting capacity needed for energy reserves and market lags, only about $25 \mathrm{kWh}$ of usable battery capacity remain. Furthermore, the optimal battery capacities of the hybrid systems are about $40 \%$ smaller than those of the BESS.

HyReK-Sell generates the highest profit regardless of the scenario, while HyReK-PtH always generates slightly higher profits than the BESS.

The battery capacity has a higher influence on the NPV than the SoC setpoints. Larger than optimal battery capacities reduce the NPV moderately in line with the additional investment and maintenance costs caused by the larger battery capacities. Smaller than optimal battery capacities result in a sharp drop in NPV, as these systems have little to no usable SoC range and therefore oscillate between buying and selling electric energy.

Even though the DoF may seem small, their strategic use improves the NPV by about $50 \mathrm{k} €$.

In the literature, the possible benefits of the DoF and $\mathrm{PtH}$ were already well-studied. The effect of battery capacity sizing was not studied to this extent before. Our results show that in the literature, battery capacities are usually higher than the optimal configuration identified here. This reduces the economic value of the system.

This study showed that providing FCR with a hybrid system has economic benefits and that battery sizing is an important topic. Future work includes the incorporation of a precise ageing model. It is also possible to determine the optimal values more precisely with more computational effort. Also, a sensitivity analysis could identify important dependencies on the assumptions.

\section{CRediT authorship contribution statement}

Uli Schlachter: Conceptualization, Methodology, Software, Investigation, Writing - Original Draft. Alena Worschech: Conceptualization, Methodology, Investigation, Writing - Original Draft. Theys Diekmann: Conceptualization, Writing - Review \& Editing, Supervision, Project administration. Benedikt Hanke: Conceptualization, Writing - Review \& Editing, Supervision, Funding 
acquisition. Karsten von Maydell: Conceptualization, Writing - Review \& Editing, Supervision, Funding acquisition.

\section{Acknowledgements}

This work has been funded by the German Federal Ministry for Economic Affairs and Energy (BMWi) under the grant no. 03ET6147C and is part of the project "HyReK 2.0 - Hybrid Regulating Power Station".

\section{References}

[1] European Commission, A clean planet for all: A European strategic long-term vision for a prosperous, modern, competitive and climate neutral economy (11 2018).

URL https://eur-lex.europa.eu/legal-content/EN/ALL/ ?uri=CELEX : 52018DC0773

[2] Council of European Union, Commission regulation (EU) 2017/1485, Official Journal of the European Union (2017). URL http: //data.europa.eu/eli/reg/2017/1485/oj

[3] 50hertz, Amprion, Tennet, and Transnet BW, Präqualifikationsverfahren für Regelreserveanbieter (FCR, aFRR, mFRR) in Deutschland ("PQ-Bedingungen"), available on http:// regelleistung.net (May 2019).

[4] M. Hiremath, K. Derendorf, T. Vogt, Comparative life cycle assessment of battery storage systems for stationary applications, Environmental Science \& Technology 49 (8) (2015) 4825-4833. doi:10.1021/es504572q.

[5] P. Stenzel, J. Linssen, M. Robinius, D. Stolten, V. Gottke, H. Teschner, A. Velten, F. Schäfer, Energiespeicher, BWK: das Energie-Fachmagazin 71 (6) (2019) 33-48.

[6] J. Fleer, S. Zurmühlen, J. Meyer, J. Badeda, P. Stenzel, J.-F. Hake, D. U. Sauer, Price development and bidding strategies for battery energy storage systems on the primary control reserve market, Energy Procedia 135 (2017) 143-157, IRES 2017. doi:10.1016/j.egypro.2017.09.497.

[7] C. Schäfer, PRL Preise 2019: Prognose weiter fallend, accessed on: 06.03.2020 (2019).

URL https://www.regelleistung-online.de/prl-preise2019/

[8] A. Oudalov, D. Chartouni, C. Ohler, Optimizing a battery energy storage system for primary frequency control, IEEE Transactions on Power Systems 22 (3) (2007) 1259-1266. doi:10.1109/TPWRS.2007.901459.

[9] M. Sandelic, D.-I. Stroe, F. Iov, Battery storage-based frequency containment reserves in large wind penetrated scenarios: A practical approach to sizing, Energies 11 (2018) 1-19. doi:10.3390/en11113065.

[10] J. Jomaux, A. Latiers, E. D. Jaeger, Cost-based dimensioning of battery energy storage and energy management system for frequency containment reserves provision, in: 2015 IEEE Power Energy Society General Meeting, 2015, pp. 1-5. doi:10.1109/PESGM.2015.7285849.

[11] S. Melo, U. Brand, T. Vogt, J. Telle, F. Schuldt, K. Maydell, Primary frequency control provided by hybrid battery storage and power-to-heat system, Applied Energy 233-234 (2019) 220231. doi:10.1016/j.apenergy.2018.09.177.

[12] A. Oudalov, D. Chartouni, C. Ohler, G. Linhofer, Value analysis of battery energy storage applications in power systems, in: PSCE 2006, IEEE PES, 2006, pp. 2206-2211. doi:10.1109/PSCE.2006.296284.

[13] F. Braeuer, J. Rominger, R. McKenna, W. Fichtner, Battery storage systems: An economic model-based analysis of parallel revenue streams and general implications for industry, Applied Energy 239 (2019) 1424-1440. doi:10.1016/j.apenergy.2019.01.050.
[14] B. Feron, A. Monti, Domestic battery and power-to-heat storage for self-consumption and provision of primary control reserve, in: PSCC, 2018, pp. 1-6. doi:10.23919/PSCC.2018.8449023.

[15] G. Angenendt, S. Zurmühlen, J. Figgener, K.-P. Kairies, D. U. Sauer, Providing frequency control reserve with photovoltaic battery energy storage systems and power-to-heat coupling, Energy 194 (2020) 1-15. doi:10.1016/j.energy.2020.116923.

[16] A. Zeh, M. Müller, M. Naumann, H. C. Hesse, A. Jossen, R. Witzmann, Fundamentals of using battery energy storage systems to provide primary control reserves in germany, Batteries 2 (3) (2016) 1-32. doi:10.3390/batteries2030029.

[17] R. Hollinger, L. M. Diazgranados, C. Wittwer, B. Engel, Optimal provision of primary frequency control with battery systems by exploiting all degrees of freedom within regulation, Energy Procedia 99 (2016) 204-214, IRES 2016. doi:10.1016/j.egypro.2016.10.111.

[18] D. Schweer, A. Maaz, A. Moser, Optimization of frequency containment reserve provision in M5BAT hybrid battery storage, in: EEM 2016, 2016, pp. 1-5. doi:10.1109/EEM.2016.7521335.

[19] J. Fleer, P. Stenzel, Impact analysis of different operation strategies for battery energy storage systems providing primary control reserve, Journal of Energy Storage 8 (2016) 320-338. doi:10.1016/j.est.2016.02.003.

[20] J. Fleer, S. Zurmühlen, J. Badeda, P. Stenzel, J.-F. Hake, D. U. Sauer, Model-based economic assessment of stationary battery systems providing primary control reserve, Energy Procedia 99 (2016) 11-24, IRES 2016. doi:10.1016/j.egypro.2016.10.093.

[21] J. Marchgraber, W. Gawlik, G. Wailzer, Reducing socmanagement and losses of battery energy storage systems during provision of frequency containment reserve, Journal of Energy Storage 27 (2020) 101107. doi:10.1016/j.est.2019.101107.

[22] S. Henninger, M. Schroeder, J. Jaeger, Combining frequency containment reserves and renewable power leveling in energy storage systems, Energy Procedia 99 (2016) 147-56, IRES 2016. doi:10.1016/j.egypro.2016.10.106.

[23] J. Marchgraber, W. Gawlik, C. Alács, Modellierung und Simulation von Batteriespeichern bei der Erbringung von Primärregelleistung, Elektrotechnik und Informationstechnik 136 (1) (2019) 3-11. doi:10.1007/s00502-019-0704-1.

[24] J. Engels, B. Claessens, G. Deconinck, Techno-economic analysis and optimal control of battery storage for frequency control services, applied to the german market, CoRR abs/1903.04251 (2019). arXiv:1903.04251. URL http://arxiv.org/abs/1903.04251

[25] H. Hesse, M. Schimpe, D. Kucevic, A. Jossen, Lithium-ion battery storage for the grid-a review of stationary battery storage system design tailored for applications in modern power grids, Energies 10 (12) (2017) 1-42. doi:10.3390/en10122107.

[26] T. Thien, D. Schweer, D. vom Stein, A. Moser, D. U. Sauer, Real-world operating strategy and sensitivity analysis of frequency containment reserve provision with battery energy storage systems in the german market, Journal of Energy Storage 13 (2017) 143-163. doi:10.1016/j.est.2017.06.012.

[27] 50hertz, Amprion, Tennet, Transnet BW, Eckpunkte und Freiheitsgrade bei der Erbringung von Primärregelleistung, available on http://regelleistung.net (04 2014).

[28] The ENTSO-e TSOs, TSOs' proposal for the establishment of common and harmonised rules and processes for the exchange and procurement of balancing capacity for frequency containment reserves (FCR) in accordance with article 33 of commission regulation (EU) 2017/2195 establishing a guideline on electricity balancing (04 2018).

[29] EPEX SPOT SE, Annual report 2018, accessed on: 06.03.2020 (2019)

URL https://www.epexspot.com/sites/default/files/ download_center_files/EPEX_RA_WEB2-compressed.pdf

[30] ENTSO-E, [Press Release] Continuing frequency deviation in the continental european power system originating in serbia/kosovo: Political solution urgently needed in addition to technical., accessed on: 07.04.2020 (2018).

URL https://www. entsoe.eu/news/2018/03/06/press- 
release-continuing-frequency-deviation-in-the-

continental-european-power-system-originating-in-

serbia-kosovo-political-solution-urgently-needed-in-

addition-to-technical/

[31] M. Sterner, I. Stadler, Energiespeicher - Bedarf, Technologien, Integration, Springer Vieweg, 2014. doi:10.1007/978-3642-37380-0.

[32] J. Vetter, P. Novák, M. Wagner, C. Veit, K.-C. Möller, J. Besenhard, M. Winter, M. Wohlfahrt-Mehrens, C. Vogler, A. Hammouche, Ageing mechanisms in lithium-ion batteries, Journal of Power Sources 147 (1) (2005) 269-281. doi:10.1016/j.jpowsour.2005.01.006.

[33] C. Pellinger, T. Schmid, Verbundforschungsvorhaben Merit Order der Energiespeicherung im Jahr 2030 - Teil 2: Technoökonomische Analyse Funktionaler Energiespeicher, Tech. rep., FfE e.V. (2016).

[34] E. Thorbergsson, V. Knap, M. Swierczynski, D. Stroe, R. Teodorescu, Primary frequency regulation with li-ion battery based energy storage system - evaluation and comparison of different control strategies, in: Intelec 2013, 2013, pp. 1-6.

URL https://www.vde-verlag.de/proceedings-en/ 453500029.html 